\title{
Finite Time Output Feedback Attitude Tracking Control for Rigid Body Based on Extended State Observer
}

\author{
Meng Duan (iD) ${ }^{1,2}$ and Yingmin Jia $\mathbb{D}^{1}$ \\ ${ }^{1}$ The Seventh Research Division and the Center for Information and Control, Beihang University, Beijing, China \\ ${ }^{2}$ School of Mathematical Sciences, Beihang University, Beijing, China \\ Correspondence should be addressed to Yingmin Jia; ymjia@buaa.edu.cn
}

Received 7 May 2020; Accepted 8 June 2020; Published 26 July 2020

Guest Editor: Jing $\mathrm{Na}$

Copyright (c) 2020 Meng Duan and Yingmin Jia. This is an open access article distributed under the Creative Commons Attribution License, which permits unrestricted use, distribution, and reproduction in any medium, provided the original work is properly cited.

In this paper, the attitude tracking control problem of output feedback is investigated. A finite time extended state observer (FTESO) is designed through the homogeneous Lyapunov method to estimate the virtual angular velocity and total disturbances. Based on these estimated states, a finite time attitude tracking controller is developed. The numerical simulations are given to illustrate the effectiveness of the proposed control scheme.

\section{Introduction}

Recently, the issue of attitude control of rigid body has received more and more attention due to its wide practical applications such as spacecraft, robotics, and maglev planar motor [1-3]. Also, the complex nonlinearity of the attitude dynamics raises potential value in theory. A great deal of control methods have been proposed to deal with the attitude control problems.

In [3], two time-varying terminal sliding mode attitude control algorithms were designed to deal with the system uncertainties and external disturbances. In [4], an adaptive regulation technique was proposed to reject the disturbances whose frequency is unknown. In [5], another adaptive attitude tracking control was implemented for the stability of a rigid spacecraft with time-varying inertia components. In [6], a backstepping method was used to design the robust attitude control for the agile satellite.

Various attitude control algorithms above need the information of full states including attitudes and angular velocities for controller design. However, in actual engineering applications, due to energy cost, weight, and other factors, an angular velocity sensor cannot be installed in the mechanical systems, or the angular velocity sensors fail to work. Thus, the design of output feedback attitude control in which just attitude measurements are available is required.

Many great research studies have been proposed to deal with the problem of output feedback attitude control. These methods can be divided into two strategies: the passivitybased controller and the observer-based controller. In [7], the passivity of attitude dynamics represented by quaternion was first proved and a passivity-based attitude control was proposed to guarantee the stability of rigid body attitude. This work was further researched in [8]; the passivity of attitude dynamics represented by modified Rodrigues parameters was proven. In [9], an auxiliary dynamical system was derived to compensate the unknown angular velocities. In [10], a passivity filter which adopted attitude Euler angles was introduced; compared to other passivity-based controllers, the proposed output feedback controller can deal with external disturbances.

The examples of observer-based attitude controller can be found in [11-14]. In [11], a finite time observer was proposed through geometric homogeneity and Lyapunov methods; a virtual angular velocity is introduced in this method. In [12], a continuous finite time angular velocity observer was designed, but the settling time could not be given. In [13], an observer was designed by adding a power integrator, thus the settling 
time can be estimated and the disturbances could be rejected by this method. In [14], a continuous terminal sliding mode observer was designed.

However, the u-modeled system dynamics and external disturbances are also the difficulties in the attitude control design. In [15-17], the unknown dynamics and external disturbances are addressed by simple structure estimators for nonlinear systems. Extended state observer (ESO) is another method to compensate these problems. The main characteristic of ESO is that all disturbances and uncertainties can be regarded as total disturbances. The linear ESO (LESO) was introduced in [18] and the structure of ESO became more concise and simple, thus ESO is preferred by engineering applications whose plants are too complex [19-21].

Inspired by [22, 23], a FTESO is introduced for the attitude control without angular velocity in this paper. The main contributions of this article are listed as follows:

Compared with other observer-based controllers, the presented control scheme can estimate angular velocity and disturbances of system simultaneously. The disturbances of the attitude system can be compensated by the outputs of proposed FTESO when the attitude controller is designed.

A finite time attitude controller which combined with FTESO is designed; it is an improvement of [11]. Due to the introduction of ESO, the proposed control framework has higher control accuracy and faster response.

This article is organized as follows. In Section 2, system dynamical models, some definitions, and lemmas are shown; the proposed FTESO-based controller is shown in Section 3; simulations are given in Section 4 and Section 5 shows the conclusions and future work.

\section{System Models and Preliminaries}

2.1. Equations of Attitude Kinematics and Dynamics. In this section, attitude system equations of a rigid body, which include attitude kinematics and dynamics, are presented.

The modified Rodrigues parameters (MRPs) are introduced to express the attitude of the rigid body; the equations of attitude kinematics are given as follows:

$$
\dot{\sigma}=T(\sigma) \omega,
$$

where $\sigma=\left[\sigma_{1}, \sigma_{2}, \sigma_{3}\right]^{T} \in \mathbf{R}^{3}$ is the MRPs which denotes the rotation from the body frame to the inertial frame and $\omega=$ $\left[\omega_{1}, \omega_{2}, \omega_{3}\right]^{T} \in \mathbf{R}^{3}$ is the angular velocity of the rigid body expressed in the body frame. The matrix $T(\sigma)$ is defined as follows:

$$
T(\sigma)=\frac{1}{4}\left[\left(1-\sigma^{T} \sigma\right) I_{3 \times 3}+2 \sigma^{\times}+2 \sigma \sigma^{T}\right],
$$

where the operator $\sigma^{\times}$represents the skew symmetric matrix of vector $\sigma$ and has the following form:

$$
\sigma^{\times}=\left[\begin{array}{ccc}
0 & -\sigma_{3} & \sigma_{2} \\
\sigma_{3} & 0 & -\sigma_{1} \\
-\sigma_{2} & \sigma_{1} & 0
\end{array}\right] .
$$

The attitude dynamics of rigid body is modeled as:

$$
J \dot{\omega}=-\omega^{\times} J \omega+\tau+d,
$$

where $\tau=\left[\tau_{1}, \tau_{2}, \tau_{3}\right]^{T} \in \mathbf{R}^{3}$ denotes the control torque, $d=$ $\left[d_{1}, d_{2}, d_{3}\right]^{T} \in \mathbf{R}^{3}$ denotes the external disturbances applied to the rigid body, and $J \in \mathbf{R}^{3 \times 3}$ denotes the inertial matrix of the rigid body which is symmetric.

Denote that $x_{1}=\sigma, x_{2}=\dot{\sigma}, x_{2}$ is the first time derivative of the attitude MRPs and is introduced as the virtual angular velocity, then equations (1) and (3) can be transformed to the following equations:

$$
\left\{\begin{array}{l}
\dot{x}_{1}=x_{2}, \\
\dot{x}_{2}=f\left(x_{1}, x_{2}\right)+g\left(x_{1}\right) \tau+d^{*}(t),
\end{array}\right.
$$

where $f\left(x_{1}, x_{2}\right)=\dot{T} P x_{2}-T J^{-1}\left(P x_{2}\right)^{\times} J P x_{2}$ is defined as system function, $P=T^{-1}(x), \quad g\left(x_{1}\right)=T J^{-1}$, and $d^{*}(t)=g\left(x_{1}\right) d$ are the external disturbances in system equation (4).

2.2. Definitions and Lemmas. For simplicity, some definitions about vector $x=\left[x_{1}, x_{2}, \ldots, x_{n}\right]^{T} \in \mathbf{R}^{n}$ are given:

$$
\begin{aligned}
x^{\alpha} & =\left[x_{1}^{\alpha}, x_{2}^{\alpha}, \ldots, x_{n}^{\alpha}\right]^{T}, \\
\operatorname{sign}(x) & =\left[\operatorname{sign}\left(x_{1}\right), \operatorname{sign}\left(x_{2}\right), \ldots, \operatorname{sign}\left(x_{n}\right)\right]^{T}, \\
\operatorname{diag}(x) & =\operatorname{diag}\left(x_{1}, x_{2}, \ldots, x_{n}\right),
\end{aligned}
$$

where $\operatorname{sign}(\cdot)$ represents the signum function and $\operatorname{diag}(\cdot)$ represents the diagonal matrix.

Lemma 1 (see [24]). For any $x \in \mathbf{R}, y \in \mathbf{R}, a>0, b>0$, $|x|^{a}|y|^{b} \leq c|x|^{a+b} /(a+b)+a|y|^{a+b} /(a+b)$.

Lemma 2 (see [25]). For any $x_{i} \in \mathbf{R}, i=1,2, \ldots, n$, $r \in(0,1], \quad\left(\sum_{i=1}^{n}\left|x_{i}\right|\right)^{r} \leq \sum_{i=1}^{n}\left|x_{i}\right|^{r} \leq n^{1-r}\left(\sum_{i=1}^{n}\left|x_{i}\right|\right)^{r}$.

Lemma 3 (see [22]). Consider the system like equation (4); assume that a Lyapunov function $V(x)$ is defined on a neighbourhood of $U \subset \mathbf{R}^{n}$ of the origin, and

$$
\dot{V}(x)+l_{1} V(x)^{m}+l_{2} V(x)^{n}<0, \quad x \in N \backslash\{0\},
$$

where $m \geq 1, n \in(0,1)$ and $l_{1}>0, l_{2}>0$. Then, the system is locally finite time stable, and the $V(x)$ which starts from $U$ can arrive at $V(x) \equiv 0$ in finite time $T$ :

$$
T \leq \frac{1}{l_{2}(1-n)}\left|V_{0}\right|^{1-n} F\left(1, \frac{1-n}{m-n}, 1+\frac{1-n}{m-n},-\frac{l_{1}}{l_{2}}\left|V_{0}\right|^{m-n}\right),
$$

where $V_{0}$ is the starting value of $V(x)$, and $F(\cdot)$ denotes the Gaussian hypergeometric function. 


\section{Main Results}

3.1. Finite Time Extended State Observer Design. In this section, only the attitude MRPs $\sigma$ is the available information; an ESO which ensures finite time convergence is presented to estimate the virtual angular velocity $x_{2}$ and the external disturbances $d^{*}(t)$.

Designate the external disturbances $d^{*}(t)$ as an extended state $x_{3}$; the system in equation (4) can be transformed to a new third-order dynamics equation which is shown in the following formulas:

$$
\left\{\begin{array}{l}
\dot{x}_{1}=x_{2} \\
\dot{x}_{2}=x_{3}+f\left(x_{1}, x_{2}\right)+g\left(x_{1}\right) \tau \\
\dot{x}_{3}=w(t)
\end{array}\right.
$$

where $w(t)=\dot{d}^{*}(t)$, and the following assumption is given.

Assumption 1. The external disturbances $d^{*}(t)$ are unknown but continuously differential and bounded; its first time derivative $w(t)$ is also unknown and bounded; the following inequalities hold:

$$
d^{*}(t) \leq \overline{d^{*}}, \quad w(t) \leq \bar{w},
$$

where $\bar{d}^{*}$ and $\bar{w}$ represent the upper bound of $d^{*}(t)$ and $w(t)$, respectively.

Let $z_{1}, z_{2}$, and $z_{3}$ represent the estimates of $x_{1}, x_{2}$, and $x_{3}$ in equation (7), respectively, then the FTESO is designed as follows:

$$
\left\{\begin{aligned}
\dot{z}_{1}= & z_{2}+\rho\left|\epsilon_{1}\right|^{\alpha_{1}} \operatorname{sign}\left(\epsilon_{1}\right)+\rho\left|\epsilon_{1}\right|^{\beta_{1}} \operatorname{sign}\left(\epsilon_{1}\right)+\theta_{1} \operatorname{sign}\left(\epsilon_{1}\right), \\
\dot{z}_{2}= & z_{3}+f\left(x_{1}, z_{2}\right)+g\left(x_{1}\right) \tau+\rho^{2}\left|\epsilon_{1}\right|^{\alpha_{2}} \operatorname{sign}\left(\epsilon_{1}\right) \\
& +\rho^{2}\left|\epsilon_{1}\right|^{\beta_{2}} \operatorname{sign}\left(\epsilon_{1}\right)+\theta_{2} \operatorname{sign}\left(\epsilon_{1}\right) \\
\dot{z}_{3}= & \rho^{3}\left|\epsilon_{1}\right|^{\alpha_{3}} \operatorname{sign}\left(\epsilon_{1}\right)+\rho^{3}\left|\epsilon_{1}\right|^{\beta_{3}} \operatorname{sign}\left(\epsilon_{1}\right)+\theta_{3} \operatorname{sign}\left(\epsilon_{1}\right),
\end{aligned}\right.
$$

where $\epsilon_{1}=x_{1}-z_{1}$ represents the estimation error of attitude MRPs $x_{1}, \quad 0<\rho<+\infty, \quad 0.5<\alpha_{1}<1, \quad \alpha_{2}=2 \alpha_{1}-1$, $\alpha_{3}=3 \alpha_{1}-2, \beta_{1}=1 / \alpha_{1}$, and $\beta_{2}=1 / \beta_{1}+\beta_{1}-1, \beta_{2}=2 / \beta_{1}+$ $\beta_{1}-2$, and $\theta_{1}, \theta_{2}$, and $\theta_{3}$ are the parameters to deal with the external disturbances.

Let $\epsilon_{2}=x_{2}-z_{2}, \epsilon_{3}=x_{3}-z_{3}$, and the error dynamical equations of the proposed FTESO is expressed as:

$$
\left\{\begin{aligned}
\dot{\epsilon}_{1}= & \epsilon_{2}-\rho\left|\epsilon_{1}\right|^{\alpha_{1}} \operatorname{sign}\left(\epsilon_{1}\right)-\rho\left|\epsilon_{1}\right|^{\beta_{1}} \operatorname{sign}\left(\epsilon_{1}\right)-\theta_{1} \operatorname{sign}\left(\epsilon_{1}\right), \\
\dot{\epsilon}_{2}= & \epsilon_{3}+f\left(x_{1}, x_{2}\right)-f\left(x_{1}, z_{2}\right)-\rho^{2}\left|\epsilon_{1}\right|^{\alpha_{2}} \operatorname{sign}\left(\epsilon_{1}\right) \\
& -\rho^{2}\left|\epsilon_{1}\right|^{\beta_{2}} \operatorname{sign}\left(\epsilon_{1}\right)-\theta_{2} \operatorname{sign}\left(\epsilon_{1}\right), \\
\dot{\epsilon}_{3}= & \omega(t)-\rho^{3}\left|\epsilon_{1}\right|^{\alpha_{3}} \operatorname{sign}\left(\epsilon_{1}\right)-\rho^{3}\left|\epsilon_{1}\right|^{\beta_{3}} \operatorname{sign}\left(\epsilon_{1}\right)-\theta_{3} \operatorname{sign}\left(\epsilon_{1}\right) .
\end{aligned}\right.
$$

The following compact set is constructed for any constant $\Delta$ :

$$
\Omega_{1}=\left\{\left(x_{1}, x_{2}, z_{1}, z_{2}\right) \mid\left\|x_{1}\right\| \leq \Delta,\left\|x_{2}\right\| \leq \Delta,\left\|z_{1}\right\| \leq \Delta,\left\|z_{2}\right\| \leq \Delta\right\} .
$$

This compact set can be easily obtained by designing a proper desired attitude reference trajectory. If the set $\Omega_{1}$ is established, there will exist a positive constant $\lambda$ such that $\left\|f\left(x_{1}, x_{2}\right)-f\left(x_{1}, z_{2}\right)\right\| \leq \lambda\left|\epsilon_{2}\right|$.

The stability properties of the extended state observer is presented in the following theorem.

Theorem 1. Consider the error dynamical system given by equation (10), where $0.5<\alpha_{1}<1, \alpha_{2}=2 \alpha_{1}-1, \alpha_{3}=3 \alpha_{1}-2$, $\beta_{1}=1 / \alpha_{1}, \beta_{2}=1 / \beta_{1}+\beta_{1}-1$, and $\beta_{2}=2 / \beta_{1}+\beta_{1}-2$; if the compact set $\Omega_{1}$ holds, $\rho, \theta_{1}, \theta_{2}$, and $\theta_{3}$ are sufficiently large, then the estimation errors would converge to a residual region in finite time.

Proof. Consider the error dynamics in equation (10); ignore the terms $\rho\left|\epsilon_{1}\right|^{\beta_{1}} \operatorname{sign}\left(\epsilon_{1}\right) \operatorname{sign}\left(\epsilon_{1}\right)-\theta_{1} \operatorname{sign}\left(\epsilon_{1}\right)$, $-\rho^{2}\left|\epsilon_{1}\right|^{\beta_{2}} \operatorname{sign}\left(\epsilon_{1}\right)-\theta_{2} \operatorname{sign}\left(\epsilon_{1}\right)$, and $w(t)-\rho^{3}\left|\epsilon_{1}\right|^{\beta_{3}} \operatorname{sign}\left(\epsilon_{1}\right)$ $-\theta_{3} \operatorname{sign}\left(\epsilon_{1}\right)$, so equation (10) can be reduced to the following form:

$$
\left\{\begin{array}{l}
\dot{\epsilon}_{1}=\epsilon_{2}-\rho\left|\epsilon_{1}\right|^{\alpha_{1}} \operatorname{sign}\left(\epsilon_{1}\right) \\
\dot{\epsilon}_{2}=\epsilon_{3}+f\left(x_{1}, x_{2}\right)-f\left(x_{1}, z_{2}\right)-\rho^{2}\left|\epsilon_{1}\right|^{\alpha_{2}} \operatorname{sign}\left(\epsilon_{1}\right), \\
\dot{\epsilon}_{3}=-\rho^{3}\left|\epsilon_{1}\right|^{\alpha_{3}} \operatorname{sign}\left(\epsilon_{1}\right) .
\end{array}\right.
$$

It can be acquired that equation (12) is homogeneous of degree $\left(\alpha_{1}-1\right)<0$ with respect to the weight $\left(1, \alpha_{1}, 2 \alpha_{1}-1\right)$.

The Lyapunov function is proposed as follows:

$$
V_{\alpha}\left(\zeta_{1}, \zeta_{2}, \zeta_{3}\right)=\zeta^{T} P \zeta
$$

where $\zeta=\left[\zeta_{1}, \zeta_{2}, \zeta_{3}\right]^{T}=\left[\left|\epsilon_{1}\right|^{1 / \varphi} \operatorname{sign}\left(\epsilon_{1}\right),\left|\epsilon_{2}\right|^{1 /\left(\varphi \alpha_{1}\right)} \operatorname{sign}\left(\epsilon_{2}\right)\right.$, $\left.\left|\epsilon_{3}\right|^{1 /\left(\varphi\left(2 \alpha_{1}-1\right)\right)} \operatorname{sign}\left(\epsilon_{3}\right)^{T}\right], 0.5<\varphi=\alpha_{1}<1, P$ denotes a positive definite symmetric matrix. Make $f_{\alpha}$ represent the vector field of system equation (12); it can be obtained that $V_{\alpha}(\zeta)$ and $L_{f_{\alpha}} V_{\alpha}(\zeta)$ are homogeneous of degrees $2 / \varphi$ and $2 / \varphi+$ $\alpha_{1}-1$ with the same weights in system equation (12), respectively.

According to $[26,27]$, the inequality is obtained as follows:

$$
L_{f_{\alpha}} V_{\alpha}(\zeta) \leq \mu_{1}(\alpha, \theta)\left(V_{a}(\zeta)\right)^{\gamma_{1}}
$$

where $\quad \mu_{1}=-\max _{\left\{y: V_{\alpha}(y)=1\right\}} L_{f_{a}} V_{\alpha(y)}, \quad \gamma_{1}=1+\left(\alpha_{1} \varphi / 2\right)-$ $\left(\alpha_{1} \varphi / 2\right) \leq 1$, and $\lim _{\alpha_{1}} \longrightarrow{ }_{1} \mu_{1} \geq \rho / \lambda_{\max }(P)$.

Same as the previous analysis, ignore the terms $\epsilon_{2}-\rho\left|\epsilon_{1}\right|^{\alpha_{1}} \operatorname{sign}\left(\epsilon_{1}\right)-\theta_{1} \operatorname{sign}\left(\epsilon_{1}\right)$,

$\epsilon_{3}+f\left(x_{1}, x_{2}\right)-f\left(x_{1}, z_{2}\right)-\rho^{2}\left|\epsilon_{1}\right|^{\alpha_{2}} \operatorname{sign}\left(\epsilon_{1}\right)-\theta_{2} \operatorname{sign}\left(\epsilon_{1}\right)$, and $\omega(t)-\rho^{3}\left|\epsilon_{1}\right|^{\alpha_{3}} \operatorname{sign}\left(\epsilon_{1}\right)-\theta_{3} \operatorname{sign}\left(\epsilon_{1}\right)$. The other homogeneous system can be got from equation (10):

$$
\left\{\begin{array}{l}
\dot{\epsilon}_{1}=-\rho\left|\epsilon_{1}\right|^{\beta_{1}} \operatorname{sign}\left(\epsilon_{1}\right), \\
\dot{\epsilon}_{2}=-\rho^{2}\left|\epsilon_{1}\right|^{\beta_{2}} \operatorname{sign}\left(\epsilon_{1}\right), \\
\dot{\epsilon}_{3}=-\rho^{3}\left|\epsilon_{1}\right|^{\beta_{3}} \operatorname{sign}\left(\epsilon_{1}\right) .
\end{array}\right.
$$


Similarly, the system of equation (15) is homogeneous of degree $\left(\beta_{1}-1\right)>0$ with respect to the weight $\left(1, \alpha_{1}, 2 \alpha_{1}-1\right)$.

Define the same Lyapunov function as equation (13) as follows:

$$
V_{\beta}\left(\zeta_{1}, \zeta_{2}, \zeta_{3}\right)=\zeta^{T} P \zeta
$$

where $\zeta=\left[\zeta_{1}, \zeta_{2}, \zeta_{3}\right]^{T}=\left[\left|\epsilon_{1}\right|^{1 / \varphi} \operatorname{sign}\left(\epsilon_{1}\right),\left|\epsilon_{2}\right|^{1 /\left(\varphi \alpha_{1}\right)} \operatorname{sign}\left(\epsilon_{2}\right)\right.$, $\left.\left|\epsilon_{3}\right|^{1 /\left(\varphi\left(2 \alpha_{1}-1\right)\right)} \operatorname{sign}\left(\epsilon_{3}\right)\right]^{T}, 0.5<\varphi=\alpha_{1}<1, P$ denotes a positive definite symmetric matrix. Make $f_{\beta}$ represent the vector field of system equation (15); it can be obtained that $V_{\beta}(\zeta)$ and $L_{f_{\beta}} V_{\beta}(\zeta)$ are homogeneous of degrees $2 / \varphi$ and $2 / \varphi+$ $\beta_{1}-1$ with the same weights in system equation (15), respectively.
Once again the following inequality is derived according to $[26,27]$

$$
L_{f_{\beta}} V_{\beta}(\zeta) \leq \mu_{2}(\beta, \theta)\left(V_{\beta}(\zeta)\right)^{\gamma_{2}},
$$

where $\mu_{2}=-\max _{\left\{y: V_{\beta}(y)=1\right\}} L_{f_{\beta}} V_{\beta(y)}, \gamma_{2}=1+\left(\varphi / 2 \beta_{1}\right)-(\varphi / 2) \geq 1$, and $\lim _{\alpha_{1} \rightarrow 1} \mu_{2} \geq \rho / \lambda_{\max }(P)$.

Considering the error dynamics in equation (10), the following Lyapunov function is chosen as

$$
V\left(\zeta_{1}, \zeta_{2}, \zeta_{3}\right)=\zeta^{T} P \zeta
$$

where $\zeta=\left[\zeta_{1}, \zeta_{2}, \zeta_{3}\right]^{T}=\left[\left|\epsilon_{1}\right|^{1 / \varphi} \operatorname{sign}\left(\epsilon_{1}\right),\left|\epsilon_{2}\right|^{1 /\left(\varphi \alpha_{1}\right)} \operatorname{sign}\left(\epsilon_{2}\right)\right.$, $\left.\left|\epsilon_{3}\right|^{1 /\left(\varphi\left(2 \alpha_{1}-1\right)\right)} \operatorname{sign}\left(\epsilon_{3}\right)\right]^{T}, 0.5<\varphi=\alpha_{1}<1, P$ denotes a positive definite symmetric matrix same as in equations (13) and (16), then take first time derivative of $V$ :

$$
\begin{aligned}
\dot{V}(\zeta)= & L_{f_{a}} V(\zeta)+L_{f_{\beta}} V(\zeta)+2 \zeta^{T} P\left[\frac{1}{\varphi \alpha_{1}} \operatorname{diag}\left(\left|\epsilon_{2}\right|\right)^{((1 / \varphi \alpha)-1)}\left(f\left(x_{1}, x_{2}\right)-f\left(x_{1}, z_{2}\right)\right)\right. \\
& +2 \zeta^{T} P\left[\begin{array}{c}
\operatorname{diag}\left(\left|\epsilon_{1}\right|\right)^{((1 / \varphi \alpha)-1)-\theta_{1}} \frac{0}{\varphi} \operatorname{sign}\left(\epsilon_{1}\right) \\
\operatorname{diag}\left(\left|\epsilon_{2}\right|\right) \\
\operatorname{diag}\left(\left|\epsilon_{3}\right|\right)^{\left(\left(1 / \varphi \alpha_{1}\right)-1\right)-\frac{\theta_{2}}{\varphi \alpha_{1}} \operatorname{sign}\left(\epsilon_{2}\right)}
\end{array}\right] .
\end{aligned}
$$

Substituting equations (14) and (17) into equation (19), the following formula can be got:

$$
\begin{aligned}
& \dot{V}(\zeta) \leq-\mu_{1}\left(V_{\alpha}(\zeta)\right)^{\gamma_{1}}-\mu_{2}\left(V_{\beta}(\zeta)\right)^{\gamma_{2}}+2 \zeta^{T} P\left[\begin{array}{c}
0 \\
\frac{1}{\varphi \alpha_{1}} \operatorname{diag}\left(\left|\epsilon_{2}\right|\right)^{\left(\left(1 / \varphi \alpha_{1}\right)-1\right)}\left(f\left(x_{1}, x_{2}\right)-f\left(x_{1}, z_{2}\right)\right) \\
0
\end{array}\right] \\
& +2 \zeta^{T} P\left[\begin{array}{c}
\operatorname{diag}\left(\left|\epsilon_{1}\right|\right)^{\left(\left(1 / \varphi \alpha_{1}\right)-1\right) \frac{-\theta_{1}}{\varphi} \operatorname{sign}\left(\epsilon_{1}\right)} \\
\operatorname{diag}\left(\left|\epsilon_{2}\right|\right)^{\left(\left(1 / \varphi \alpha_{1}\right)-1\right)} \frac{-\theta_{2}}{\varphi \alpha_{1}} \operatorname{sign}\left(\epsilon_{2}\right) \\
\operatorname{diag}\left(\left|\epsilon_{3}\right|\right)^{\left(\left(1 / 2 \varphi \alpha_{1}\right)-1\right) \frac{w(t)-\theta_{3}}{\varphi} \operatorname{sign}\left(\epsilon_{3}\right)}
\end{array}\right] \leq-\mu_{1}\left(V_{\alpha}(\zeta)\right)^{\gamma_{1}}-\mu_{2}\left(V_{\beta}(\zeta)\right)^{\gamma_{2}} \\
& +\frac{2 \lambda \lambda_{\max }(P)\|\zeta\|\left(\sum_{i=1}^{3}\left|\varepsilon_{2 i}\right|^{\left(1 / \varphi \alpha_{1}\right)-1}\right)\left(\sum_{i=1}^{3}\left|\varepsilon_{2 i}\right|\right)}{\varphi \alpha_{1}} \\
& +\frac{2 \theta_{1} \lambda_{\max }(P)\|\zeta\|\left(\sum_{i=1}^{3}\left|\epsilon_{1 i}\right|^{(1 / \varphi)-1}\right)}{\varphi}+\frac{2 \theta_{2} \lambda_{\max }(P)\|\zeta\|\left(\sum_{i=1}^{3}\left|\epsilon_{2 i}\right|^{\left(1 / \varphi \alpha_{1}\right)-1}\right)}{\varphi \alpha_{1}} \\
& +\frac{2\left(\theta_{3}+\bar{w}\right) \lambda_{\max }(P)\|\zeta\|\left(\sum_{i=1}^{3}\left|\epsilon_{2 i}\right|^{\left(1 / \varphi\left(2 \alpha_{1}-1\right)\right)-1}\right)}{\varphi\left(2 \alpha_{1}-1\right)},
\end{aligned}
$$


where $\lambda_{\max }(P)$ refers to the maximum eigenvalue of matrix $P$.

The following inequality is derived according to Lemma 1 :

$$
\left|\epsilon_{2 j}\right|^{\left(1 / \varphi \alpha_{1}\right)-1}\left(\sum_{i=1}^{3}\left|\epsilon_{2 i}\right|\right) \leq 3\left(1 / \varphi \alpha_{1}\right)\left|\epsilon_{2 j}\right|^{(1 / \varphi \alpha)}+\varphi \alpha_{1}\left(\sum_{i=1}^{3}\left|\epsilon_{2 i}\right|^{\left(1 / \varphi \alpha_{1}\right)}\right)
$$

where $j=1,2,3$, and the following result is got:

$$
\left(\sum_{i=1}^{3}\left|\epsilon_{2 i}\right|^{\left(1 / \varphi \alpha_{1}\right)-1}\right)\left(\sum_{i=1}^{3}\left|\epsilon_{2 i}\right|\right) \leq 3\left(\sum_{i=1}^{3}\left|\epsilon_{2 i}\right|^{\left(1 / \varphi \alpha_{1}\right)}\right) \leq 3 \sqrt{3}\left\|\zeta_{2}\right\| \leq 3 \sqrt{3}\|\zeta\|
$$

2:

The following inequality is derived according to Lemma

$$
\sum_{i=1}^{3}\left|\epsilon_{2 i}\right|^{(1 / \varphi \alpha)-1}=\sum_{i=1}^{3}\left|\epsilon_{2 i}\right|^{(1 / \varphi \alpha)(1-\varphi \alpha)} \leq 3^{\varphi \alpha_{1}}\left(\sum_{i=1}^{3}\left|\zeta_{2 i}\right|\right)^{1-\varphi \alpha_{1}} \leq 3^{\varphi \alpha_{1}}\left(\sqrt{3}\left\|\zeta_{2}\right\|\right)^{1-\varphi \alpha_{1}} \leq 3^{\left(1+\varphi \alpha_{1}\right) / 2}\|\zeta\|^{1-\varphi \alpha_{1}}
$$

The following inequalities can be derived in the same way according to Lemma 2 :

$$
\begin{gathered}
\sum_{i=1}^{3}\left|\epsilon_{1 i}\right|^{(1 / \varphi)-1} \leq 3^{(1 / \varphi) / 2}\|\zeta\|^{1-\varphi}, \\
\sum_{i=1}^{3}\left|\epsilon_{1 i}\right|^{\left(1 / \varphi\left(2 \alpha_{1}-1\right)\right)-1} \leq 3^{\left(1 / \varphi\left(2 \alpha_{1}-1\right)\right) / 2}\|\zeta\|^{1-\varphi\left(2 \alpha_{1}-1\right)} .
\end{gathered}
$$

$$
\begin{aligned}
\dot{V}(\zeta) \leq & -\mu_{1}\left(V_{\alpha}(\zeta)\right)^{\gamma_{1}}-\mu_{2}\left(V_{\beta}(\zeta)\right)^{\gamma_{2}}=+\frac{6 \sqrt{2} \lambda \lambda_{\max }(P)\|\zeta\|^{2}}{\varphi \alpha_{1}}+\frac{2 \times 3^{\left(1+\varphi \alpha_{1} / 2\right)} \theta_{1} \lambda_{\max }(P)\|\zeta\|^{2-\varphi}}{\varphi} \\
& +\frac{2 \times 3^{(1+\varphi / 2)} \theta_{2} \lambda_{\max }(P)\|\zeta\|^{2-\varphi \alpha_{1}}}{\varphi \alpha_{1}}+\frac{2 \times 3^{1+\varphi\left(2 \alpha_{1}-1\right) / 2}\left(\theta_{3}+\bar{w}\right) \lambda_{\max }(P)\|\zeta\|^{2-\varphi\left(2 \alpha_{1}-1\right)}}{\varphi\left(2 \alpha_{1}-1\right)} \leq-\mu_{1} V^{\gamma_{1}}-\mu_{2} V^{\gamma_{2}}+\mu_{3} V+\mu_{4} V^{1-(\varphi-2)} \\
& +\mu_{5} V^{1-\left(\varphi \alpha_{1} / 2\right)}+\mu_{6} V^{1-\left(\varphi\left(2 \alpha_{1}-1\right)\right)}
\end{aligned}
$$

So, substituting equations (22)-(25) into the inequality equation (20), the following inequality can be got: where

$$
\begin{aligned}
& \mu_{3}=\frac{6 \sqrt{2} \lambda \lambda_{\max }(P)}{\varphi \lambda_{\min }(P)}, \\
& \mu_{4}=\frac{2 \times 3^{\left(1+\varphi \alpha_{1} / 2\right)} \theta_{1} \lambda_{\max }(P)}{\varphi \lambda_{\min }(P)^{1-(\varphi / 2)}}, \\
& \mu_{5}=\frac{2 \times 3^{(1+\varphi / 2)} \theta_{2} \lambda_{\max }(P)}{\varphi \lambda_{\min }(P)^{1-(\varphi / 2)}}, \\
& \mu_{6}=\frac{2 \times 3^{\left(1+\varphi / 2 \alpha_{1} / 2\right)}\left(\theta_{3}+\bar{w}\right) \lambda_{\max }(P)}{\varphi \lambda_{\min }(P)^{1-\left(\varphi \alpha_{1} / 2\right)}} .
\end{aligned}
$$

Two cases are discussed in further analysis.

Case 1. $V \geq 1$.

Consider the parameters in equation (26):

$$
\begin{aligned}
& 0<\left(1-\frac{\varphi}{2}\right)<1<\gamma_{2}, \\
& 0<\left(1-\frac{\varphi \alpha_{1}}{2}\right)<1<\gamma_{2}, \\
& 0<\left(1-\frac{\varphi\left(2 \alpha_{1}-1\right)}{2}\right)<1<\gamma_{2} .
\end{aligned}
$$

So, simplify equation (26) to the following formula: 


$$
\dot{V} \leq-\mu_{1} V^{\gamma_{1}}-\mu_{7} V^{\gamma_{2}}
$$

where $\mu_{7}=\mu_{2}-\mu_{3}-\mu_{4}-\mu_{5}-\mu_{6}$; select the parameter $\rho$ sufficiently large to ensure $\mu_{7}>0$; according to Lemma 3, the Lyapunov function $V$ will converge to $V(\zeta) \equiv 1$ in finite time $t_{1}$.

$$
t_{1}<\frac{1}{\mu_{1}\left(1-\gamma_{1}\right)} V_{(0)}^{1-\gamma_{1}} F\left(1, \frac{1-\gamma_{1}}{\gamma_{2}-\gamma_{1}}, 1+\frac{1-\gamma_{1}}{\gamma_{2}-\gamma_{1}},-\frac{\mu_{7}}{\mu_{1}} V_{(0)}^{\gamma_{2}-\gamma_{1}}\right)
$$

where $V_{(0)}$ denotes the initial value of $V$.

Case 2. $V<1$. In this case, equation (26) can be simplified to

$$
\begin{aligned}
\dot{V} \leq & -\mu_{1} V^{\gamma_{1}}-\mu_{2} V^{\gamma_{2}}+\mu_{3} V^{\gamma_{1}}+\left(\mu_{4}+\mu_{5}+\mu_{6}\right) V^{1-(\varphi / 2)} \leq-\mu_{1}\left(1-\rho_{0}\right) V^{\gamma_{1}}+\mu_{3} V^{\gamma_{1}}+\left(\mu_{4}+\mu_{5}+\mu_{6}\right) V^{1-(q / 2)} \\
& -\mu_{1} \rho_{0} V^{\gamma_{1}}-\mu_{2} V^{\gamma_{2}} \leq-\left[\left(\mu_{1}\left(1-\rho_{0}\right)-\mu_{3}\right) V^{\gamma_{1}-1+(q / 2)}-\left(\mu_{4}+\mu_{5}+\mu_{6}\right)\right] V^{1-(q / 2)}-\mu_{1} \rho_{0} V^{\gamma_{1}}-\mu_{2} V^{\gamma_{2}},
\end{aligned}
$$

where the parameter $\rho_{0}$ is selected to ensure that $0<\rho_{0}<1$.

From inequality equation (30), when $\left(\mu_{1}\left(1-\rho_{0}\right)-\mu_{3}\right) V^{\gamma_{1}-1+(\varphi / 2)}-\left(\mu_{4}+\mu_{5}+\mu_{6}\right) \geq 0$, the Lyapunov function $V$ will converge to the residual region $\Omega_{2}$ in finite time $T_{1}=t_{1}+t_{2}$ :

$\Omega_{2}=\left\{V \mid V<\left(\frac{\mu_{4}+\mu_{5}+\mu_{6}}{\mu_{1}\left(1-\rho_{0}\right)-\mu_{3}}\right)^{\left(2 / 2 \gamma_{1}-2+\varphi\right)}\right\}$,
$t_{2}<\frac{1}{\mu_{1} \rho_{0}\left(1-\gamma_{1}\right)} V_{\left(t_{1}\right)}^{1-\gamma_{1}} \times F\left(1, \frac{1-\gamma_{1}}{\gamma_{2}-\gamma_{1}}, 1+\frac{1-\gamma_{1}}{\gamma_{2}-\gamma_{1}},-\frac{\mu_{2}}{\mu_{1} \rho_{0}} V_{\left(t_{1}\right)}^{\gamma_{2}-\gamma_{1}}\right)$, where $V_{\left(t_{1}\right)}$ represents the value of $V$ at time $t_{1}$. The estimation error can be written as

$$
\Omega_{3}=\left\{\zeta \mid \frac{1}{\sqrt{\lambda_{\min }(\mathbf{P})}}\left(\frac{\mu_{4}+\mu_{5}+\mu_{6}}{\mu_{1}\left(1-\rho_{0}\right)-\mu_{3}}\right)^{\left(1 / 2 \gamma_{1}-2+\varphi\right)}\right\} .
$$

In next step, the parameters $\theta_{1}, \theta_{2}$, and $\theta_{3}$ will be analyzed to ensure $\left(\epsilon_{1}, \epsilon_{2}, \epsilon_{3}\right)$ converge to zeros within finite time.

Define the following Lyapunov function:

$$
V_{\epsilon_{1}}\left(\epsilon_{1}\right)=\frac{1}{2} \epsilon_{1}^{T} \epsilon_{1} \text {. }
$$

Take time derivative of $V_{\epsilon_{1}}$ :

$$
\dot{V}_{\epsilon_{1}}=\epsilon_{1}^{T} \dot{\epsilon}_{1}=\epsilon_{1}^{T} \epsilon_{2}-\rho \sum_{i=1}^{3}\left|\epsilon_{1 i}\right|^{\alpha_{1}+1}-\rho \sum_{i=1}^{3}\left|\epsilon_{1 i}\right|^{\beta_{1}+1}-\theta_{1} \sum_{i=1}^{3}\left|\epsilon_{1}\right| \leq-\rho\left\|\epsilon_{1}\right\|^{\alpha_{1}+1 / 2}-\rho\left\|\epsilon_{1}\right\|^{\beta_{1}+1 / 2}-\left(\theta_{1}-\left\|\epsilon_{2}\right\|\right)\left\|\epsilon_{1}\right\| .
$$

$$
\dot{V}_{\epsilon_{1}} \leq-2^{\alpha_{1}+1 / 2} V_{\epsilon_{1}}^{\alpha_{1}+1 / 2}-2^{\beta_{1}+1 / 2} \rho V_{\epsilon_{1}}^{\beta_{1}+1 / 2} .
$$

According to equation (33), $\epsilon_{2}$ will converge to the bounded region $\Omega_{3}$, and then selecting the parameter $\theta_{1}>\Omega_{3}$, the inequality equation (35) can be simplified to
So, $\epsilon_{1}$ can converge to zeros within time $T_{2}=T_{1}+t_{3}$ :

$$
t_{3}<\frac{1}{2^{\alpha_{1}-1 / 2}}\left(1-\alpha_{1}\right) \times F\left(1, \frac{1-\alpha_{1}}{\beta_{1}-\alpha_{1}}, 1+\frac{1-\alpha_{1}}{\beta_{1}-\alpha_{1}},-2^{\beta_{1}-\alpha_{1} / 2} V\left(e_{1}\right)_{T_{1}}^{\beta_{1}-\alpha_{1}}\right),
$$

where $V_{\epsilon_{1}\left(T_{1}\right)}$ is the value of $V_{\epsilon_{1}}$ at time $t_{1}$.

So, $\epsilon_{1}=\epsilon_{1}=0$ is got; according to [28], discontinuous terms include that $\operatorname{sign}\left(\epsilon_{1}\right)$ can be considered as equivalent input; the error dynamical system equation (10) can be reduced to

$$
\left\{\begin{array}{l}
0=\epsilon_{2}-\theta_{1} \operatorname{sign}\left(\epsilon_{1}\right) \\
\dot{\epsilon}_{2}=\epsilon_{3}+\lambda\left|\epsilon_{2}\right|-\theta_{2} \operatorname{sign}\left(\epsilon_{1}\right) \\
\dot{\epsilon}_{3}=\omega(t)-\theta_{3} \operatorname{sign}\left(\epsilon_{1}\right)
\end{array}\right.
$$


It can be derived that $\operatorname{sign}\left(\epsilon_{2}\right)=\operatorname{sign}\left(\epsilon_{1}\right)$. Define the Lyapunov function $V_{\epsilon_{2}}\left(\epsilon_{2}\right)=(1 / 2) \epsilon_{2}^{T} \epsilon_{2}$ and $V_{\epsilon_{3}}\left(\epsilon_{3}\right)=(1 / 2) \epsilon_{3}^{T} \epsilon_{3}$. Analyze these two Lyapunov functions like we did before; it can be easily obtained that, let $\theta_{2}>\Omega_{3}$ and $\theta_{1}>\bar{w}$, the estimation errors $\epsilon_{2}$ and $\epsilon_{3}$ will converge to zeros within finite time $T_{3}=T_{2}+t_{4}$ and $T_{4}=T_{3}+t_{5}$, respectively [11]:

$$
\begin{aligned}
& t_{4}<\frac{2^{(1 / 2)}}{\left|\theta_{2}-\Omega_{3}\right|}\left|V_{\epsilon_{2}\left(T_{2}\right)}\right|^{(1 / 2)}, \\
& t_{5}<\frac{2^{(1 / 2)}}{\left|\theta_{3}-\bar{w}\right|}\left|V_{\epsilon_{3}\left(T_{3}\right)}\right|^{(1 / 2)},
\end{aligned}
$$

where $V_{\epsilon_{2}\left(T_{2}\right)}$ denotes the value of $V_{\epsilon_{2}}$ at time $T_{2}$ and $V_{\epsilon_{3}\left(T_{3}\right)}$ denotes the value of $V_{\epsilon_{3}}$ at time $T_{3}$.
At this point, the proof is complete.

3.2. Finite Time Attitude Controller Design Based on FTESO. In this section, inspired by Zou [11], a finite time controller is introduced to stabilize the rigid body to the desired attitude. Because only the attitude measurement is available, the estimation of virtual angular velocity $z_{2}$ and external disturbances $z_{3}$ are utilized in the design procedure.

Define $\xi=\eta^{1 / v}-\eta_{d}^{1 / v}$ and $e=x_{1}-\sigma_{d}$, where $\eta=z_{2}-\dot{\sigma}_{d}$, $\eta_{d}=-k_{1} e^{\nu}, 1 / 2<\nu=\nu_{1} / \nu_{2}<1$, and $\nu_{1}$ and $\nu_{2}$ are positive odd integers.

The proposed finite time attitude controller is shown as follow:

$$
\tau=g^{-1}\left(-k_{3} \operatorname{sign}(\xi)-k_{2} \xi^{2 v-1}-z_{3}-f\left(x_{1}, z_{2}\right)+\ddot{q}_{d}-\rho^{2}\left|\epsilon_{1}\right|^{\alpha_{2}} \operatorname{sign}\left(\epsilon_{1}\right)-\rho^{2}\left|\epsilon_{1}\right|^{\beta_{2}} \operatorname{sign}\left(\epsilon_{1}\right)-\theta_{2} \operatorname{sign}\left(\epsilon_{1}\right)\right) .
$$

Theorem 2. Consider the system expressed in equation (4); if finite time control torque in equation (40) combined with

$$
\begin{aligned}
& V_{c}=V_{0}+\sum_{i=1}^{3} V_{i}, \\
& V_{0}=\frac{1}{2} e^{T} e .
\end{aligned}
$$

Proof. Define the Lyapunov function:

$$
V_{f}=V+V_{c},
$$

where

$V_{i}$ is defined as

$$
V_{i}=\frac{1}{(2-v) 2^{1-v} k_{1}^{1+1 / v}} \int_{\eta_{d i}}^{\eta_{i}}\left(s^{1 / v}-\eta_{d i}^{1 / v}\right)^{2-v} \mathrm{~d} s
$$

Taking time derivative of Lyapunov function $V_{f}$, the result can be got as follows:

$$
\dot{V}_{f}=\dot{V}+\dot{V}_{c}=\dot{V}+\dot{V}_{0}+\sum_{i=1}^{3} \dot{V}_{i} \leq-\mu_{1} V^{\gamma_{1}}-\mu_{2} V^{\gamma_{2}}+\mu_{3} V+\mu_{4} V^{1-\varphi / 2}+\mu_{5} V^{1-\left(\varphi \alpha_{1} / 2\right)}+\mu_{6} V^{1-\varphi\left(2 \alpha_{1}-1\right) / 2}+\dot{V}_{0}+\sum_{i=1}^{3} \dot{V}_{i}
$$

In this section, just the terms $\dot{V}_{0}$ and $\sum_{i=1}^{3} \dot{V}_{i}$ need to be derived. After tedious derivation, it could be obtained that

$$
\begin{aligned}
& \dot{V}_{0} \leq-k_{1} e^{T} e^{v}+\sum_{i=1}^{3} \frac{2^{1-v}}{1+v}\left(\left|e_{i}\right|^{1+v}+v\left|\xi_{i}\right|^{1+v}\right)+e^{T} \epsilon_{2}, \\
& \dot{V}_{i} \leq \frac{1}{k_{1}}\left|\epsilon_{2 i}\right|\left|\xi_{i}\right|+\frac{\left(k_{1}+2^{1-\nu}(1+v)\right)\left|\xi_{i}\right|^{1+v}}{k_{1}(1+v)}+\frac{v\left|e_{i}\right|^{1+v}}{1+v}+\frac{1}{(2-v) 2^{1-v} k_{1}^{1+1 / v}} \xi_{i}^{2-v} \bar{\tau}_{i},
\end{aligned}
$$

where $\bar{\tau}_{i}=\partial \eta_{i} / \partial t$.

Substitute the control torque equation (40) into equation (47). The $\dot{V}_{i}$ can be derived as follows:

$$
\dot{V}_{i} \leq \frac{1}{k_{1}}\left|\epsilon_{2 i}\right|\left|\xi_{i}\right|+\frac{\left(k_{1}+2^{1-\nu}(1+\nu)\right)\left|\xi_{i}\right|^{1+\nu}}{k_{1}(1+\nu)}+\frac{v\left|e_{i}\right|^{1+\nu}}{1+v}+\frac{-k_{2}}{(2-v) 2^{1-\nu} k_{1}^{1+1 / v}} \xi_{i}^{1+\nu}+\frac{-k_{3}}{(2-v) 2^{1-\nu} k_{1}^{1+1 / v}}\left|\xi_{i}\right|^{2-\nu} .
$$


Combining equation (48) with equations (42) and (46),

$$
\begin{aligned}
\dot{V}_{c} \leq & -\left(k_{1}-\frac{2^{1-v}+\nu}{1+\nu}\right) \sum_{i=1}^{3}\left|e_{i}\right|^{1+v}+e^{T} \zeta_{2} \\
& +\sum_{i=1}^{3} \frac{\left|\zeta_{2 i}\right|\left|\zeta_{i}\right|}{k_{1}}-\left(\frac{k_{2}}{(2-v) 2^{1-v} k_{1}^{1+1 / v}}-\frac{k_{1}+2^{1-\nu}(1+v)}{k_{1}(1+v)}\right) \times \sum_{i=1}^{3}\left|\xi_{i}\right|^{1+v}+\frac{-k_{3}}{(2-v) 2^{1-v} k_{1}^{1+1 / v}} \sum_{i=1}^{3}\left|\xi_{i}\right|^{2-\nu} .
\end{aligned}
$$

Choose $k_{3}>0$ and let

$k_{1} \geq \frac{2^{1-\nu}+v}{1+v}+k_{4} k_{2} \geq(2-v) 2^{1-v} k_{1}^{1+1 / v}\left(\frac{k_{1}+2^{1-\nu}(1+v)}{k_{1}(1+v)}+k_{4}\right)$, where $k_{4}$ is the positive constant. The following inequality is obtained:

$$
\dot{V}_{c} \leq-k_{3} \sum_{i=1}^{3}\left|e_{i}\right|^{1+v}-k_{3} \sum_{i=1}^{3}\left|\xi_{i}\right|^{1+v}+e^{T} \epsilon_{2}+\sum_{i=1}^{3} \frac{\left|\epsilon_{2 i}\right|\left|\xi_{i}\right|}{k_{1}} \leq c_{1}\left(\sum_{i=1}^{3} e_{i}^{2}+\sum_{i=1}^{3} \xi_{i}^{2}\right)
$$

where $c_{1}=\max \left\{1 / 2,1 /(2-v) k_{1}^{1+1 / v}\right\}$.

Notice that

$$
V_{i} \leq \frac{1}{(2-v) 2^{1-v} k_{1}^{1+1 / v}}\left|\eta_{i}-\eta_{d i} \| \xi_{i}\right|^{2-\nu} \leq \frac{1}{(2-v) k_{1}^{1+1 / v}}\left|\xi_{i}\right|^{2}
$$

Hence, the following inequality is obtained:

$$
V_{c}=V_{0}+\sum_{i=1}^{3} V_{i} \leq c_{2}\left(\sum_{i=1}^{3} \epsilon_{i}^{2}+\sum_{i=1}^{3} \xi_{i}^{2}\right)
$$

where $c_{2}=\max \left\{1 / 2,1 /(2-v) k_{1}^{1+1 / v}\right\}$.

According to Lemma 2:

$$
\dot{V}_{c} \leq-c_{3} V^{(1+v) / 2}+e^{T} \epsilon_{2}+\sum_{i=1}^{3} \frac{\left|\epsilon_{2 i}\right|\left|\xi_{i}\right|}{k_{1}}
$$

where $c_{3}=k_{3} / c_{2}^{(1+v) / 2}$.

From equations (45) and (54),

$$
\begin{aligned}
\dot{V}_{f}= & \dot{V}+\dot{V}_{c}=\dot{V}+\dot{V}_{0}+\sum_{i=1}^{3} \dot{V}_{i} \leq-\mu_{1} V^{\gamma_{1}}-\mu_{2} V^{\gamma_{2}}+\mu_{3} V \\
& +\mu_{4} V^{1-(\varphi / 2)}+\mu_{5} V^{1-\varphi \alpha_{1} / 2}+\mu_{6} V^{1-\varphi\left(2 \alpha_{1}-1\right) / 2}-c_{2} V^{(1+v) / 2}+e^{T} \epsilon_{2}+\sum_{i=1}^{3} \frac{\left|\epsilon_{2 i}\right|\left|\xi_{i}\right|}{k_{1}} .
\end{aligned}
$$

According to Theorem 1, $\epsilon(t)$ would converge to zeros in finite time, so equation (55) becomes

$$
\dot{V}_{f}=\dot{V}_{c} \leq-c_{2} V_{c}^{(1+v) / 2} \text {. }
$$

Because $0<(1+v) / 2<1$, the tracking errors $e$ converge to zeros in finite time [11].

The proof is completed.
Remark 1. In practical situations, due to the absence of certain parameters, the system function $f\left(x_{1}, x_{2}\right)$ can not be calculated accurately. To deal with this problem, the system function $f\left(x_{1}, x_{2}\right)$ and external disturbances $d^{*}(t)$ can be regarded as total disturbances $d_{\text {total }}(t)$. The control algorithm proposed in this article can be written as

$$
\left\{\begin{array}{l}
\dot{z}_{1}=z_{2}+\rho\left|\epsilon_{1}\right|^{\alpha_{1}} \operatorname{sign}\left(\epsilon_{1}\right)+\rho\left|\epsilon_{1}\right|^{\beta_{1}} \operatorname{sign}\left(\epsilon_{1}\right)+\theta_{1} \operatorname{sign}\left(\epsilon_{1}\right), \\
\dot{z}_{2}=z_{3}+g\left(x_{1}\right) \tau+\rho^{2}\left|\epsilon_{1}\right|^{\alpha_{2}} \operatorname{sign}\left(\epsilon_{1}\right)+\rho^{2}\left|\epsilon_{1}\right|^{\beta_{2}} \operatorname{sign}\left(\epsilon_{1}\right)+\theta_{2} \operatorname{sign}\left(\epsilon_{1}\right), \\
\dot{z}_{3}=\rho^{3}\left|\epsilon_{1}\right|^{\alpha_{3}} \operatorname{sign}\left(\epsilon_{1}\right)+\rho^{3}\left|\epsilon_{1}\right|^{\beta_{3}} \operatorname{sign}\left(\epsilon_{1}\right)+\theta_{3} \operatorname{sign}\left(\epsilon_{1}\right), \\
\tau=g^{-1}\left(-k_{3} \operatorname{sign}(\xi)-k_{2} \xi^{2 v-1}-z_{3}+\ddot{q}_{d}-\rho^{2}\left|\epsilon_{1}\right|^{\alpha_{2}} \operatorname{sign}\left(\epsilon_{1}\right)-\rho^{2}\left|\epsilon_{1}\right|^{\beta_{2}} \operatorname{sign}\left(\epsilon_{1}\right)-\theta_{2} \operatorname{sign}\left(\epsilon_{1}\right)\right) .
\end{array}\right.
$$




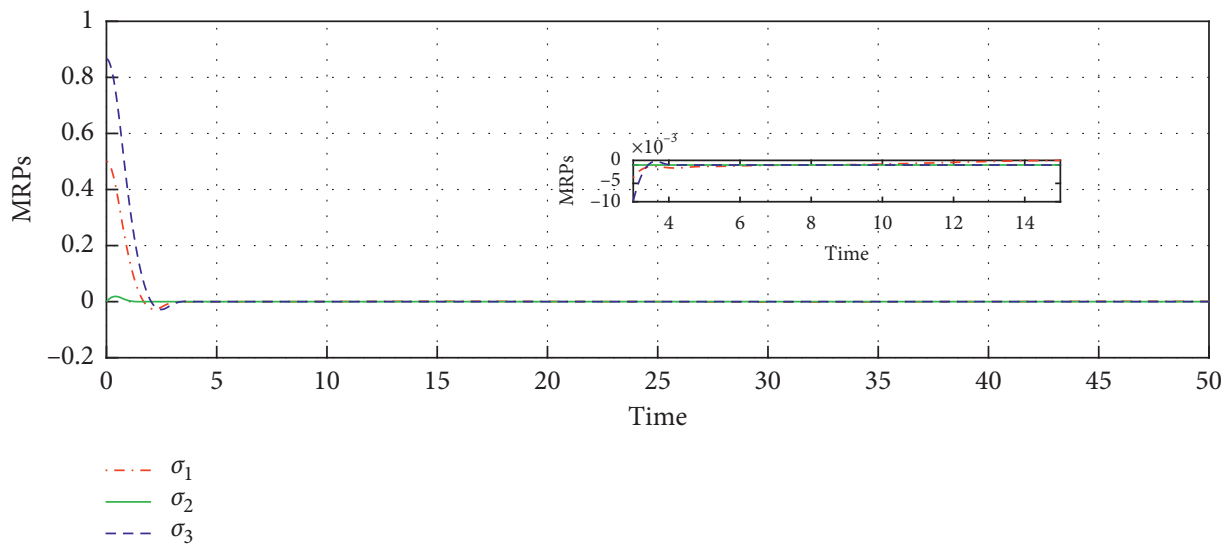

Figure 1: Tracking errors for FTESO-based controller with external disturbances.

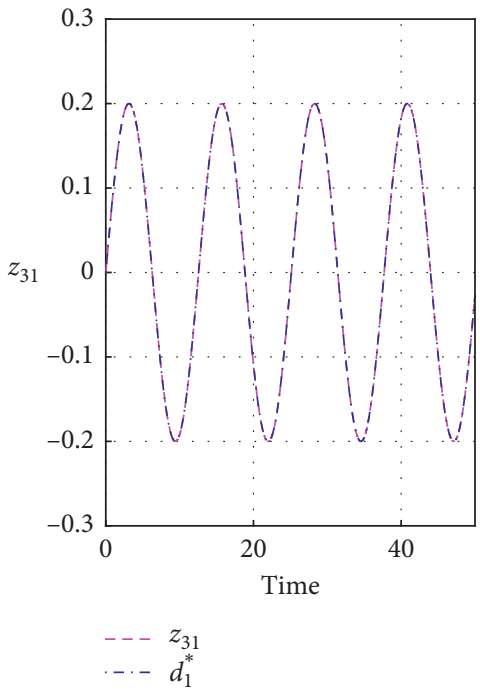

(a)

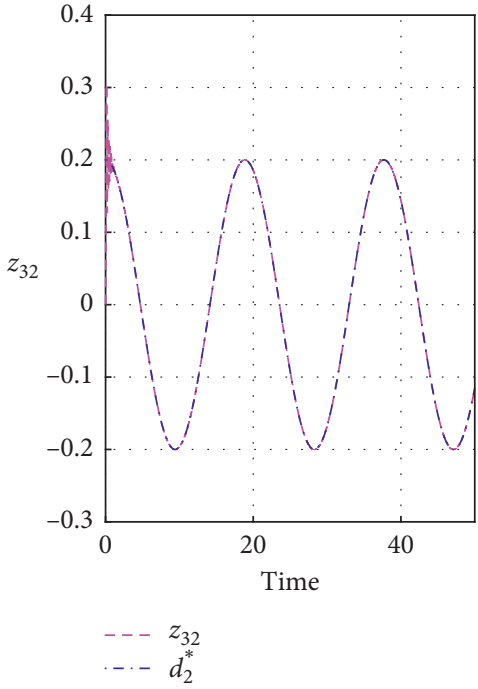

(b)

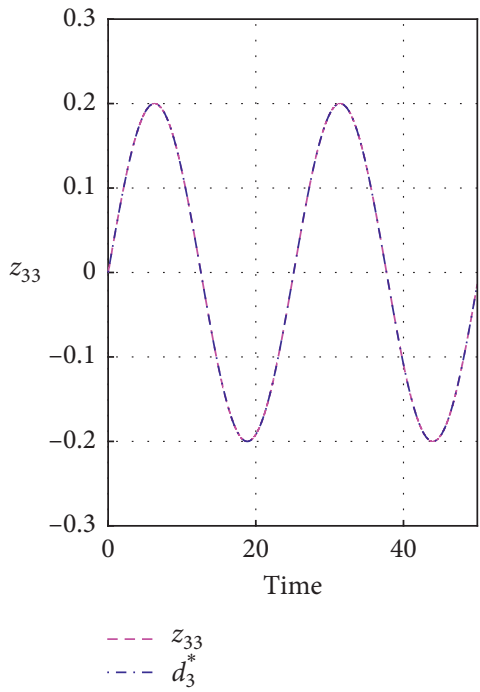

(c)

FIgURe 2: External disturbances estimate for FTESO. (a) $z_{31}$. (b) $z_{32}$. (c) $z_{33}$.

Remark 2. In order to eliminate the chattering issue caused by discontinuous terms in the observer and controller, the signum function can be replaced by the following function:

$$
\operatorname{sgmf}\left(e_{1}\right)= \begin{cases}2\left(\frac{1}{1+\exp \left(-\gamma_{o} e_{1}\right)}-\frac{1}{2}\right), & \left|e_{1}\right| \leq \delta, \\ \operatorname{sign}\left(e_{1}\right), & \left|e_{1}\right|>\delta,\end{cases}
$$

where $\gamma_{o}$ and $\delta$ are constants which can be designed.

\section{Simulation Results}

In this section, several numerical simulations are implemented to show the effectiveness of the FTESO-based control scheme proposed in this paper; meanwhile, the control scheme with general LESO and the control scheme without ESO in reference [11] are introduced as comparisons.
The initial states are designed as $x_{1}(0)=[0,0,0]^{T}$, $x_{2}(0)=[0,0,0]^{T}, z_{1}(0)=[0,0,0]^{T}, z_{2}(0)=[0,0,0]^{T}, z_{3}(0)=$ $[0,0,0]^{T}$. The desired attitude trajectory is designed as $\sigma_{d}=0.5[\cos (0.2 t), \sin (0.2 t), \sqrt{3}]^{T}$, and the external disturbances $d^{*}=0.2[\sin (0.5 t), \cos (1 / 3 t), \sin (1 / 4 t)]$. The parameters of the proposed ESO and controller are $\rho=5$, $\alpha_{1}=0.8, \theta_{1}=1, \theta_{2}=5, \theta_{3}=1, v=7 / 9, k_{1}=1.1, k_{2}=1.5$, and $k_{3}=0.01$. The inertia matrix of rigid body is chosen as

$$
J=\left[\begin{array}{ccc}
20 & 1.2 & 0.9 \\
1.2 & 17 & 1.4 \\
0.9 & 1.4 & 15
\end{array}\right] \mathrm{kgm}^{2} .
$$

The upper bound of control torque is selected as $\left|\tau_{i}\right| \leq 50 \mathrm{Nm}$, where $i=1,2,3$.

First, the proposed scheme includes that equations (9) and (40) are simulated. In this simulation, the system function $f\left(x_{1}, x_{2}\right)$ is assumed to be known; the proposed 


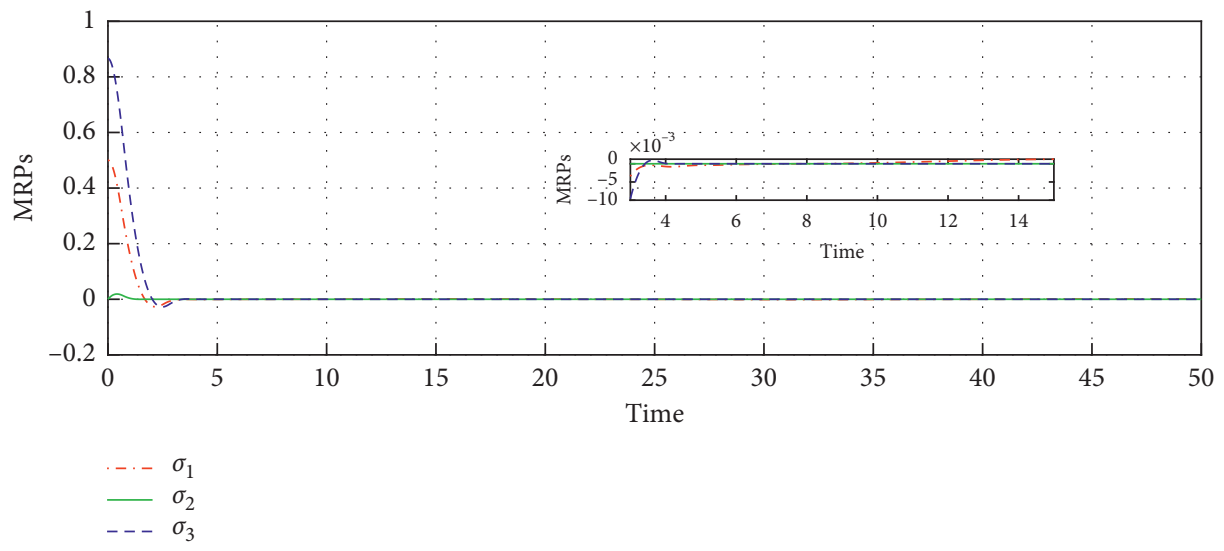

FIGURE 3: Tracking errors for the FTESO-based controller with total disturbances.

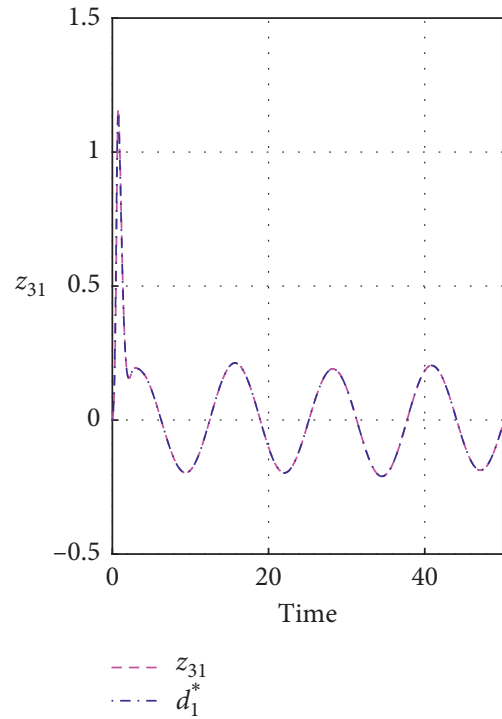

(a)

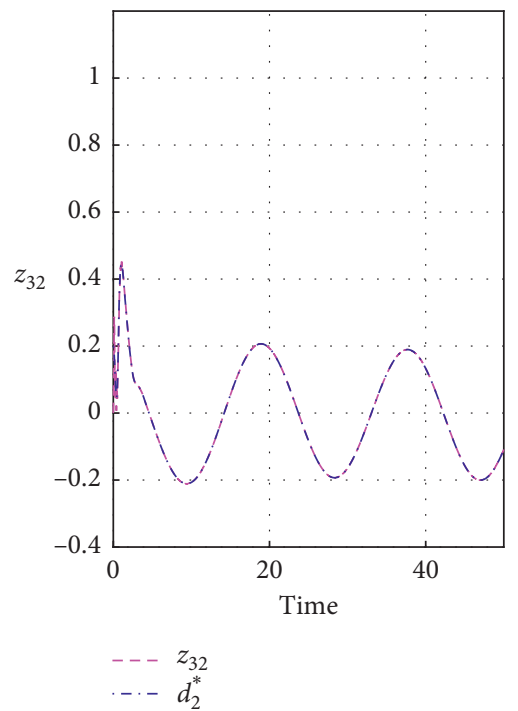

(b)

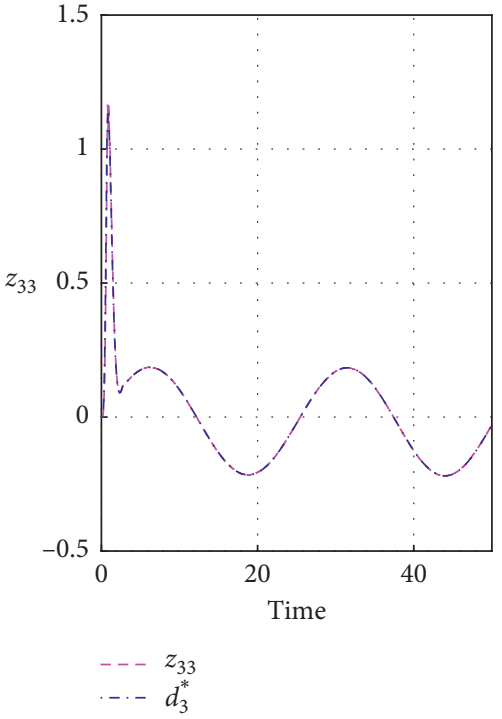

(c)

FIGURE 4: Total disturbances estimate for FTESO. (a) $z_{31}$. (b) $z_{32}$. (c) $z_{33}$.

FTESO only estimates the external disturbances $d^{*}$ and the virtual angular velocity $x_{2}$.

The results of attitude tracking errors and external disturbances estimate for the FTESO-based control scheme are shown in Figures 1 and 2. According to Figure 1, the proposed control scheme can converge the tracking errors to zeros within about 4 seconds, and the extended state $z_{3}$ can also estimate the total disturbances $d^{*}(t)$ in finite time.

Then, consider the situation that the system function $f\left(x_{1}, x_{2}\right)$ is unknown, which is regarded as total disturbances $d_{\text {total }}(t)$ together with external disturbances $d^{*}(t)$. The simulation results are shown in Figures 3 and 4 . Compared with above results, the total disturbances $d_{\text {total }}(t)$ is much larger than $d^{*}(t)$ in Figure 2, but it can be fast estimated by FTESO. Because the estimated total disturbances can be compensated in controller, even the system function $f\left(x_{1}, x_{2}\right)$ is unknown; the proposed control scheme can also converge the tracking errors to zeros within finite time. The FTESO guarantees that the two simulations in Figures 1 and 3 have the similar results.

For comparison, the introduced linear LESO is designed as follows:

$$
\left\{\begin{array}{l}
\dot{z}_{1}=z_{2}+\rho_{1} \varepsilon_{1}, \\
\dot{z}_{2}=z_{3}+f\left(x_{1}, z_{2}\right)+g\left(x_{1}\right) \tau+\rho_{1}^{2} \varepsilon_{1}, \\
\dot{z}_{3}=\rho_{1}^{3} \varepsilon_{1} .
\end{array}\right.
$$

The corresponding controller is similar to the one designed in this article:

$$
\tau=g^{-1}\left(-k_{3} \operatorname{sign}(\xi)-k_{2} \xi^{2 v-1}-z_{3}-f\left(x_{1}, z_{2}\right)+\ddot{q}_{d}-\rho_{1}^{2} \varepsilon_{1}\right) \text {. }
$$

The parameters are designed as $\rho_{1}=28, \nu=7 / 9$, $k_{1}=1.1, k_{2}=1.5$, and $k_{3}=0.01$.

The other control scheme in [11] is expressed as 


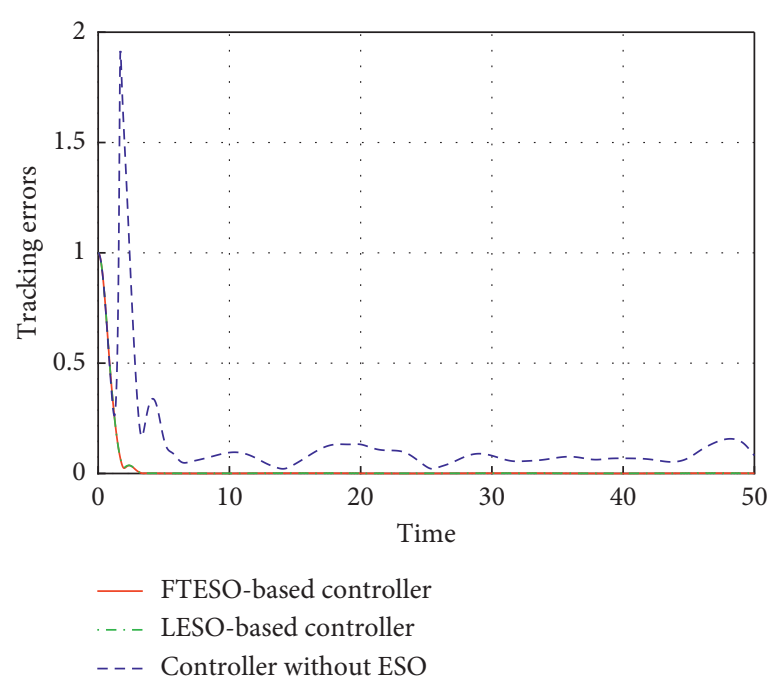

(a)

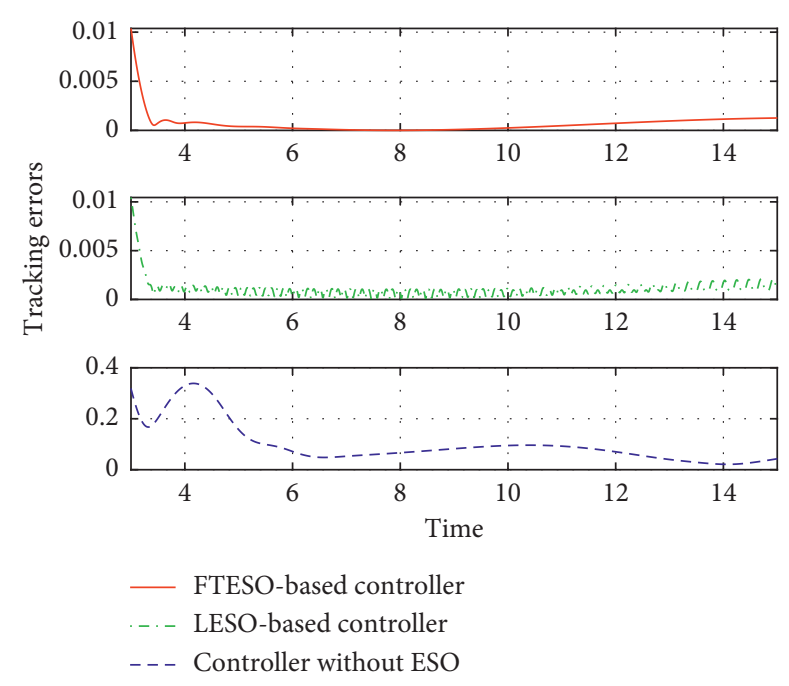

(b)

Figure 5: Tracking errors $\|e\|$ under different control schemes.

$$
\left\{\begin{array}{l}
\dot{z}_{1}=z_{2}+\theta c_{1}\left|\varepsilon_{1}\right|^{\alpha_{1}} \operatorname{sign}\left(\varepsilon_{1}\right), \\
\dot{z}_{2}=z_{3}+f\left(x_{1}, z_{2}\right)+g\left(x_{1}\right) \tau+\theta^{2} c_{2}\left|\varepsilon_{1}\right|^{\alpha_{2}} \operatorname{sign}\left(\varepsilon_{1}\right), \\
\tau=g^{-1}\left(-k_{2} \xi^{2 v-1}-f\left(x_{1}, z_{2}\right)+\ddot{q}_{d}-\theta^{2} c_{2}\left|\varepsilon_{1}\right|^{\alpha_{2}} \operatorname{sign}\left(\varepsilon_{1}\right)\right),
\end{array}\right.
$$

where the parameters are selected to be same as in [11]: $\theta=3, \quad c_{1}=1, \quad c_{2}=0.2, v=7 / 9, \quad k_{1}=1.1, \quad k_{2}=1.5$, and $k_{3}=0.01$.

The comparison results between these three attitude control schemes are shown in Figure 5. As shown in Figure 5, the proposed control scheme in this article and the control algorithm based on LESO in equations (59) and (60) can attain faster convergence than the controller in equation (61), and the proposed control scheme gets the best disturbances rejection.

\section{Conclusion}

The finite time attitude tracking control for rigid body is studied in this article; a FTESO is designed through the homogeneous Lyapunov method. The angular velocity and total disturbances can be estimated by the proposed observer. Based on this, a finite time attitude controller is introduced. Numerical simulations show that the proposed control scheme can achieve fast convergence and good disturbances rejection. Further work will be focused on reducing power cost and dealing with the input saturation problem.

\section{Data Availability}

All data generated or analyzed during this study are included within the article.

\section{Conflicts of Interest}

The authors declare that they have no conflicts of interest.

\section{Acknowledgments}

This work was supported by the National Basic Research Program of China (973 Program: 2012CB821200 and 2012CB 821201) and the NSFC (61327807, 61521091, 61520106010, and 61134005).

\section{References}

[1] M. Miyasaka and P. Berkelman, "Magnetic levitation with unlimited omnidirectional rotation range," Mechatronics, vol. 24, no. 3, pp. 252-264, 2014.

[2] Y. Hong, Y. Xu, and J. Huang, "Finite-time control for robot manipulators," Systems \& Control Letters, vol. 46, no. 4, pp. 243-253, 2002.

[3] L. Zhao and Y. Jia, "Finite-time attitude tracking control for a rigid spacecraft using time-varying terminal sliding mode techniques," International Journal of Control, vol. 88, no. 6, pp. 1150-1162, 2015.

[4] Z. Chen and J. Huang, "Attitude tracking of rigid spacecraft subject to disturbances of unknown frequencies," International Journal of Robust and Nonlinear Control, vol. 24, no. 16, pp. 2231-2242, 2014.

[5] D. Thakur, S. Srikant, and M. R. Akella, "Adaptive attitudetracking control of spacecraft with uncertain time-varying inertia parameters," Journal of Guidance, Control, and Dynamics, vol. 38, no. 1, pp. 41-52, 2015.

[6] H. Zhang and J. Fang, "Robust backstepping control for agile satellite using double-gimbal variable-speed control moment gyroscope," Journal of Guidance, Control, and Dynamics, vol. 36, no. 5, pp. 1356-1363, 2013.

[7] F. Lizarralde and J. T. Wen, "Attitude control without angular velocity measurement: a passivity approach," IEEE Transactions on Automatic Control, vol. 41, no. 3, pp. 468-472, 1996.

[8] P. Tsiotras, "Further passivity results for the attitude control problem," IEEE Transactions on Automatic Control, vol. 43, no. 11, pp. 1597-1600, 1998.

[9] A. Tayebi, "Unit quaternion-based output feedback for the attitude tracking problem," IEEE Transactions on Automatic Control, vol. 53, no. 6, pp. 1516-1520, 2008. 
[10] B. Xiao, L. Cao, and D. Ran, "Attitude exponential stabilization control of rigid bodies via disturbance observer," IEEE Transactions on Systems, Man, and Cybernetics: Systems, vol. 53, pp. 1-9, 2019.

[11] A.-M. Zou, "Finite-time output feedback attitude tracking control for rigid spacecraft," IEEE Transactions on Control Systems Technology, vol. 22, no. 1, pp. 338-345, 2014.

[12] H. Gui and G. Vukovich, "Finite-time angular velocity observers for rigid-body attitude tracking with bounded inputs," International Journal of Robust and Nonlinear Control, vol. 27, no. 1, pp. 15-38, 2017.

[13] B. Jiang, C. Li, and G. Ma, "Finite-time output feedback attitude control for spacecraft using "Adding a power integrator" technique," Aerospace Science and Technology, vol. 66, pp. 342-354, 2017.

[14] Q. Hu and B. Jiang, "Continuous finite-time attitude control for rigid spacecraft based on angular velocity observer," IEEE Transactions on Aerospace and Electronic Systems, vol. 54, no. 3, pp. 1082-1092, 2018.

[15] J. Na, B. Jing, Y. Huang, G. Gao, and C. Zhang, "Unknown system dynamics estimator for motion control of nonlinear robotic systems," IEEE Transactions on Industrial Electronics, vol. 67, no. 5, pp. 3850-3859, 2020.

[16] J. Na, J. Yang, S. Wang, G. Gao, and C. Yang, "Unknown dynamics estimator-based output-feedback control for nonlinear pure-feedback systems," IEEE Transactions on Systems, Man, and Cybernetics: Systems, vol. 53, pp. 1-12, 2019.

[17] J. Na, S. Wang, Y.-J. Liu, Y. Huang, and X. Ren, "Finite-time convergence adaptive neural network control for nonlinear servo systems," IEEE Transactions on Cybernetics, vol. 50, no. 6, pp. 2568-2579, 2020.

[18] Z. Gao, "Scaling and bandwidth parameterization based controller tuning," in Proceedings of the 2003 American Control Conference, vol. 6, pp. 4989-4996, Denver, Colorado, USA, 2003.

[19] L. Zhao, H. Cheng, and T. Wang, "Sliding mode control for a two-joint coupling nonlinear system based on extended state observer," Isa Transactions, vol. 73, pp. 130-140, 2018.

[20] Y.-j. Wu and G.-f. Li, "Adaptive disturbance compensation finite control set optimal control for pmsm systems based on sliding mode extended state observer," Mechanical Systems and Signal Processing, vol. 98, pp. 402-414, 2018.

[21] R. Madonski, M. Ramirez-Neria, M. Stanković et al., "On vibration suppression and trajectory tracking in largely uncertain torsional system: an error-based adrc approach," Mechanical Systems and Signal Processing, vol. 134, p. 106300, 2019.

[22] Q. Hu, B. Jiang, and M. I. Friswell, "Robust saturated finite time output feedback attitude stabilization for rigid spacecraft," Journal of Guidance, Control, and Dynamics, vol. 37, no. 6, pp. 1914-1929, 2014.

[23] S. Xiong, W. Wang, X. Liu, Z. Chen, and S. Wang, "A novel extended state observer," Isa Transactions, vol. 58, pp. 309317, 2015.

[24] C. Qian and W. Lin, "A continuous feedback approach to global strong stabilization of nonlinear systems," IEEE Transactions on Automatic Control, vol. 46, no. 7, pp. 10611079, 2001.

[25] Y. Shen and X. Xia, "Semi-global finite-time observers for nonlinear systems," Automatica, vol. 44, no. 12, pp. 31523156, 2008.

[26] S. P. Bhat and D. S. Bernstein, "Geometric homogeneity with applications to finite-time stability," Mathematics of Control, Signals, and Systems, vol. 17, no. 2, pp. 101-127, 2005.
[27] Y. Shen and Y. Huang, "Uniformly observable and globally lipschitzian nonlinear systems admit global finite-time observers," IEEE Transactions on Automatic Control, vol. 54, no. 11, pp. 2621-2625, 2009.

[28] I. Haskara, "On sliding mode observers via equivalent control approach," International Journal of Control, vol. 71, no. 6, pp. 1051-1067, 1998. 